\title{
Evaluación de la Incorporación de Fibra Prebiótica sobre la Viabilidad de Lactobacillus casei Impregnado en Matrices de Mora (Rubus glaucus)
}

\author{
Sneyder Rodríguez-Barona ${ }^{(1)^{*}}$, Gloria I. Giraldo ${ }^{(2)}$ y Yenni P. Zuluaga ${ }^{(3)}$ \\ (1) Universidad Nacional de Colombia, Sede Manizales, Departamento de Ingeniería Química, Campus la \\ Nubia Km 4 Vía al Magdalena, AA 127 Manizales, Colombia (e-mail: snrodriguezba@unal.edu.co) \\ (2) Universidad Nacional de Colombia, Sede Manizales, Departamento de Física y Química \\ (e-mail: gigiraldogo@unal.edu.co) \\ (3) Universidad de Caldas, Departamento de Ciencias Biológicas (e-mail: ypzp@hotmail.com) \\ ${ }^{*}$ Autor a quien debe ser dirigida la correspondencia
}

Recibido May. 8, 2015; Aceptado Jun. 11, 2015; Versión final Jun. 25, 2015, Publicado Oct. 2015

\begin{abstract}
Resumen
En este trabajo se evaluó el efecto de la fibra prebiótica en la supervivencia de la cepa Lactobacillus casei ATCC 393, incorporado en matrices de mora mediante un proceso de impregnación a vacío y posterior liofilización. Láminas de mora fueron impregnadas con soluciones isotónicas de inulina, fruto-oligosacárido (FOS), inulina-FOS y control sin prebiótico, inoculadas con $10^{9} \mathrm{UCF} / \mathrm{ml}$ del microorganismo. Las muestras impregnadas fueron liofilizadas y almacenadas a condiciones ambientales para su seguimiento. Se observó efecto favorable de la presencia de prebióticos sobre la viabilidad del microorganismo al permitir la supervivencia por más de 40 días, con recuentos superiores a $10^{6} \mathrm{UCF} / \mathrm{g}$ mientras en la muestra control fue inferior luego de 25 días. El análisis sensorial arrojó un puntaje de aceptación superior a 5 en una escala de 1 a 7. La presencia de los microorganismos incorporados en la fruta fue evidenciada mediante las observaciones con microscopía electrónica de barrido.
\end{abstract}

Palabras clave: alimentos funcionales; fruto-oligosacárido; inulina; impregnación; prebiótico; probióticos.

\section{Evaluation of the Incorporation of Prebiotic Fiber on the Viability of Lactobacillus casei Impregnated in Matrices of Andean Blackberry (Rubus glaucus)}

\begin{abstract}
This study showed the favorable effect of prebiotic fiber in the survival of Lactobacillus casei of ATCC 393, incorporated in matrices of Andean blackberry by a process of vacuum impregnation and subsequent lyophilization. Blackberry slices were impregnated with isotonic solutions of inulin, fruit - oligosaccharide (FOS) and inulin-FOS control without prebiotic inoculated with $10^{9} \mathrm{UCF} / \mathrm{ml}$ of the microorganism. The impregnated samples were lyophilized and stored at ambient conditions for monitoring. Favorable effects were observed for the presence of prebiotics to the viability of the microorganism, allowing its survival for more than 40 days, with higher counts to $106 \mathrm{UCF} / \mathrm{g}$ while in the control sample was lower after 25 days. Sensory analysis showed a higher acceptance score greater than 5 on a scale from 1 to 7 . The presence of microorganisms embedded in the fruit was evidenced by the observation with scanning electronic microscopy.
\end{abstract}

Keywords: functional food; fructo-oligosaccharides; inulin; vacuum impregnation; prebiotics; probiotics 


\section{INTRODUCCIÓN}

Los probióticos son un tipo de alimento funcional y se definen como microorganismos vivos que al ser ingeridos en cantidades suficientes, ejercen un efecto positivo en la salud, realizando acciones saludables en el organismo, entre ellas equilibrando la flora intestinal y potenciando el sistema inmunológico (Vasiljevic y Shah, 2008;Tripathi y Giri, 2014). A su vez, los prebióticos son componentes con actividad fisiológica presentes en los alimentos, resisten la digestión en el intestino delgado y son susceptibles de ser fermentados por la flora bacteriana benéfica del intestino grueso, favoreciendo el equilibrio de la flora intestinal y regulando el tránsito intestinal al favorecer el peristaltismo. Los prebióticos mejoran la adsorción de ciertos minerales y reducen los niveles de glucosa en sangre, siendo por ello aptos para el consumo en diabéticos (Roberfroid, 2000). Entre los azúcares prebióticos más comunes en alimentos se encuentran la inulina y los frutooligosacáridos. Su estructura comprende una cadena lineal de residuos de fructosa con la propiedad de aportar menor número de calorías al ser comparada con carbohidratos digeribles.

Los prebióticos mejoran la viabilidad y supervivencia de los microorganismos probióticos durante la producción y almacenamiento de los alimentos (Krasaekoopt y Watcharapoka, 2014), en tratamientos de secado se unen a las células y reducen el diferencial osmótico entre el medio interno y externo protegiéndolas de los efectos de la deshidratación (Rajam y Anandharamakrishnan, 2015). La acción sinérgica de la unión de un probiótico con un prebiótico alude al término de alimento simbiótico (Sarmiento, 2006; De Vrese y Schrezenmeir, 2008). Una amplia gama de alimentos probióticos y simbióticos presentes en el mercado son de origen lácteo (Aljewicz y Cichosz, 2015, Zapata et al., 2015). Sin embargo, alimentos de origen vegetal, como frutas y hortalizas, ofrecen una alternativa importante como vehículo de componentes fisiológicamente activos (CFA) naturales e incorporados, adecuados para cualquier consumidor (Ursachia et al., 2009). Actualmente, algunos avances tecnológicos hacen posible alterar las características estructurales de las matrices de frutas y verduras modificando sus componentes de una manera controlada (Betoret et al., 2003). En el desarrollo de alimentos funcionales a partir de alimentos estructurados, se ha implementado una de las operaciones de la ingeniería de matrices más aplicadas, la técnica de impregnación a vacío (IV) la cual puede ser aplicada como herramienta de incorporación de CFA en un producto poroso, modificando su composición para proveerlo de cualidades que incrementen su calidad nutricional (Fito et al., 2001; Gras et al., 2003; Andrés-Bello et al., 2015).

La mora de castilla en Colombia es considerada un cultivo con grandes oportunidades, tanto para el abastecimiento del mercado interno, como para exportación. Entre sus cualidades nutricionales se destaca el aporte de hidratos de carbono, minerales como calcio, hierro, potasio; vitaminas C y A y su acción antioxidante por el alto contenido de antocianinas y carotenoides (Mertz, et al., 2009).

En este sentido, la adición de microorganismos probióticos con fibra prebiótica en matrices vegetales, podría favorecer la supervivencia de los microorganismos durante el almacenamiento y consumo de los productos funcionales obtenidos y a su vez la obtención de un alimento con cualidades simbióticas. De esta manera, el presente trabajo de investigación evaluó la viabilidad de la cepa de Lactobacillus casei, incorporada en mezcla con agentes prebióticos en matrices de mora de castilla mediante la técnica de impregnación a vacío y conservadas por liofilización.

\section{MATERIALES Y MÉTODOS}

\section{Probiótico y soluciones de impregnación}

La cepa liofilizada de Lactobacillus casei ATCC 393 fue adquirida a través de una casa comercial, activada en caldo MRS (Scharlou) e incubada a $37^{\circ} \mathrm{C}$ durante 48 horas en condiciones aerobias y conservada en crioviales hasta su uso. Se trabajó con dos agentes prebióticos: inulina comercial (INU) extraída de la raíz de achicoria y fructo-oligosacárido (FOS) (marca Nutraflora® corn productos Andina). Se prepararon tres soluciones isotónicas de impregnación con los ${ }^{\circ}$ Brix de la fruta, a partir de los agentes prebióticos: inulina, FOS y una mezcla 1:1 de FOS:Inulina. Se consideró como solución control, agua estéril.

La biomasa para los ensayos fue obtenida luego de la fermentación en medio MRS (Scharlau) e incubada a $37^{\circ} \mathrm{C}$ y recuperada al final de la fase exponencial de crecimiento mediante centrifugación a $5000 \mathrm{rpm}$ (Hettich Universal 320 ) durante 15 minutos a $4^{\circ} \mathrm{C}$ y lavada con solución salina al $0.5 \%$.

\section{Preparación de las muestras}

Las moras de castilla (Rubus glaucus) fueron adquiridas a través de un proveedor de la zona rural del municipio de Manizales, Caldas, Colombia. La selección de las muestras para los ensayos se realizó según apariencia y color. Las moras empleadas en los ensayos presentaron las siguientes características: $\mathrm{pH}$ $2.95 \pm 0.01$, sólidos solubles $8.5 \pm 0.5^{\circ}$ Brix, actividad de agua $0.98 \pm 0.01$ y humedad $87.7 \pm 0.81$. 
Las muestras fueron sometidas a un proceso de higienización retirando inicialmente los peciolos, luego se sumergieron en una solución de agua e hipoclorito al $0.5 \%(\mathrm{p} / \mathrm{v})$ durante 10 minutos, se enjuagaron con suficiente agua y se cortaron en láminas de $2.02 \pm 0.1 \mathrm{~cm}$ de diámetro con $0.59 \pm 0.06 \mathrm{~cm}$ de espesor.

\section{Caracterización físico-química}

Las propiedades físicas y químicas de las muestras impregnadas y liofilizadas se determinaron por duplicado en las muestras homogeneizadas.

El contenido de sólidos en la fase líquida de las muestras fue medido a temperatura ambiente $\left(20^{\circ} \mathrm{C}\right)$ en unidades Brix usando un refractómetro (Brixco Instruments). El valor de pH fue medido con un potenciómetro (Schott-handylabpH11)). El valor de actividad de agua fue determinado usando un higrómetro de punto de rocío (Thermo-Constantern Novasina). La humedad de las muestras fue medida hasta peso constante según el método AOAC 934.06. (A.O.A.C., 1999). A las soluciones de impregnación, además de pH y ํㅜix, se les midió densidad con picnómetro y viscosidad con el uso de un viscosímetro Ubbelohde.

\section{Inoculación de soluciones de impregnación}

La biomasa recuperada fue adicionada a las soluciones de impregnación hasta una concentración de microorganismos de $10^{9} \mathrm{UFC} / \mathrm{mL}$.

\section{Proceso de Impregnación a vacío (IV)}

Las láminas de mora, debidamente identificadas, fueron pesadas y sumergidas en las soluciones vehículo, llevadas al dispositivo de impregnación a vacío (Rotavapor Buchi R-114) y sometidas a una presión de vacío de 50 mbar durante 10 minutos a temperatura ambiente $\left(20^{\circ} \mathrm{C}\right)$. Posteriormente se restableció la presión atmosférica y las muestras continuaron sumergidas 10 minutos adicionales. Durante el proceso de IV se evaluó la evolución de la masa de las muestras impregnadas. A cada una de las muestras impregnadas se le determinaron los parámetros de impregnación: $X_{M F}$ fracción volumétrica ocupada por el líquido $\left(\mathrm{m}^{3} \mathrm{SI} / \mathrm{m}^{3}\right.$ mora fresca), Xiv contenido másico de prebiótico ( $\mathrm{kg}$ prebiótico $/ \mathrm{kg}_{\text {mora }}$ impregnada) y la porosidad efectiva, $\varepsilon_{\mathrm{e}}$, a partir de las siguientes ecuaciones (Cortés et al., 2007)

$$
\begin{aligned}
& \mathrm{X}_{\mathrm{MF}}=\frac{\mathrm{M}_{\mathrm{f}}-\mathrm{M}_{\mathrm{i}}}{\rho_{\text {dis }}\left(\frac{\mathrm{M}_{\mathrm{i}}}{\rho_{\mathrm{apm}}}\right)} \\
& \mathrm{X}_{\mathrm{IV}}=\frac{\left(\mathrm{M}_{\mathrm{f}}-\mathrm{M}_{\mathrm{i}}\right)^{*} \frac{\mathrm{M}_{\text {preb }}}{100 \mathrm{ml}}}{\mathrm{M}_{\mathrm{f}}{ }^{*} \rho_{\text {dis }}}
\end{aligned}
$$

Dónde: $M_{\mathrm{f}}$ : peso final de la muestra $(\mathrm{g}), \mathrm{M}_{\mathrm{i}}$ : peso inicial de la muestra $(\mathrm{g}), \rho_{\text {dis }}$ : densidad de la solución $\left(\mathrm{g} / \mathrm{cm}^{3}\right), \rho_{\text {apm }}$ : densidad aparente de la mora, Mpreb: gramos de prebiótico

$$
\varepsilon_{\mathrm{e}}=\frac{\mathrm{X}}{(1-1 / \mathrm{r})}
$$

$\varepsilon_{\mathrm{e}}$ : Porosidad efectiva, $X$ : fracción volumétrica del líquido incorporado, $r=P_{a t m} / P_{v a c i o}$

\section{Secado y almacenamiento de la fruta}

Para su estabilización, las láminas de mora impregnadas fueron deshidratadas en un Liofilizador Génesis 25 (Virtis, modelo ADVANTAGE XL, USA). El proceso de liofilización tuvo un tiempo de duración de 48 horas, hasta obtener el ciclo completo de liofilización. Las muestras liofilizadas fueron almacenadas en bolsas metalizadas de barrera media (BOPP metalizado/Pa/Pe), empacadas a vacío y conservadas a temperatura ambiente $\left(20^{\circ} \mathrm{C}\right)$.

\section{Recuentos microbianos y evaluación de la viabilidad del microorganismo}

El recuento de unidades formadores de colonia por gramo (UFC/g) se determinó para las muestras de fruta liofilizadas mediante el procedimiento de siembra en placa, incubación y conteo sobre agar MRS, a una temperatura de $37^{\circ} \mathrm{C}$ durante $24 \mathrm{~h}$ aproximadamente. Para la incubación, las muestras liofilizadas fueron previamente rehidratadas con peptona tamponada $(0,1 \% \mathrm{p} / \mathrm{v})$ en proporción 1:9 durante una hora. Los resultados se expresaron en unidades logarítmicas $\left(\log _{10} \mathrm{UFC} / \mathrm{g}\right)$. Los recuentos en cada caso fueron 
realizados por triplicado. Para el recuento y preparación de las muestras sólidas se siguieron las recomendaciones dadas por Champagne y colaboradores (Champagne et al., 2011).

\section{Análisis microscópico}

La observación a diferentes aumentos de los microorganismos impregnados en la matriz de la fruta liofilizada se realizó en un microscopio electrónico de barrido (SEM) (Scanning Electron Microscopio marca JEOL referencia JSM 5950 LV). Luego de recubrir las muestras con oro sublimado.

\section{Evaluación sensorial}

Se realizó una prueba hedónica a 33 jueces no entrenados sobre una escala verbal de preferencia de ocho niveles, siendo 1 (me desagradada muchísimo) y 8 (me agrada muchísimo) (Anzaldúa, 1994) aplicada con el fin disminuir la subjetividad de las apreciaciones de los jueces. Para el análisis se utilizó la fruta impregnada y liofilizada, adicionada a muestras de yogurt y cereal.

\section{Análisis de datos}

En todos los casos, los datos experimentales fueron tomados por triplicado y analizados a partir de un ANOVA. Se utilizó el método LSD (Mínimas Diferencias Significativas) como método de comparaciones múltiples, con un nivel de confianza del 95\% $(p=0,05)$. El análisis de varianza fue realizado con el paquete estadístico STATGRAPHICS PLUS versión 5.1 .

\section{RESULTADOS Y DISCUSIÓN}

\section{Caracterización fisicoquímica de las soluciones de impregnación}

Las soluciones empleadas como medio para incorporar componentes activos en los poros o espacios intercelulares de matrices alimenticias, requiere cumplir con ciertas características; entre ellas, la concentración de solutos deberá ser isotónica con respecto al tejido para evitar transferencias de masa indeseables a través de las membranas y el valor de $\mathrm{pH}$ deberá estar en un rango tal que no afecte la estabilidad de los microrganismos ni de los tejidos. Por otro lado, la viscosidad de la solución deberá permitir su flujo al interior de los poros y/o espacios intracelulares, pues determina la pérdida de carga durante la entrada del líquido a los poros de la fruta (Fito y Pastor, 1994). Finalmente, la concentración de microorganismos en el líquido de impregnación deberá ser lo suficientemente alta, para lograr incorporar en número adecuado ( $>10^{6} \mathrm{UFC} / \mathrm{g}$ ) los microrganismos al interior de la fruta y conferir las características probióticas buscadas en el alimento (Betoret et al., 2012; Rêgo et al., 2013). En la tabla1 se presentan las propiedades de las soluciones de impregnación utilizadas en este trabajo.

Tabla 1. Propiedades de la soluciones de impregnación. 1: Soluciones antes de inoculación 2: Soluciones inoculadas

\begin{tabular}{|c|c|c|c|c|c|c|c|}
\hline \multicolumn{3}{|c|}{ Parámetro } & $\mathrm{pH}$ & ${ }^{\circ}$ Brix & Densidad $\left(\mathrm{g} / \mathrm{cm}^{3}\right)$ & Viscosidad (mPa.s) & $\mathrm{UFC} / \mathrm{g} \times 10^{12}$ \\
\hline \multirow{6}{*}{$\begin{array}{l}: \frac{\overline{0}}{0} \\
\frac{\bar{O}}{0} \\
\text { C. }\end{array}$} & \multirow{2}{*}{ Inulina } & 1 & $6.67 \pm 0.05$ & $8.00 \pm 0.50$ & $1053.06 \pm 0.006$ & $1.2420 \pm 0.006$ & \\
\hline & & 2 & $3.47 \pm 0.01$ & $7.90 \pm 0.68$ & $1056.63 \pm 0.013$ & $1.4258 \pm 0.013$ & 4.4 \\
\hline & \multirow{2}{*}{ FOS } & 1 & $6.35 \pm 0.04$ & $8.40 \pm 0.50$ & $1042.57 \pm 0.004$ & $1.2509 \pm 0.004$ & \\
\hline & & 2 & $3.29 \pm 0.01$ & $8.40 \pm 0.60$ & $1050.36 \pm 0.001$ & $1.3843 \pm 0.006$ & 0.9 \\
\hline & \multirow{2}{*}{ Inulina + FOS } & 1 & $6.78 \pm 0.07$ & $9.50 \pm 0.67$ & $1053.45 \pm 0.007$ & $1.3662 \pm 0.020$ & \\
\hline & & 2 & $3.49 \pm 0.01$ & $9.49 \pm 0.57$ & $1072.76 \pm 0.003$ & $1.4401 \pm 0.010$ & 1.5 \\
\hline
\end{tabular}

Los resultados mostrados en la tabla 1, resumen las propiedades de las soluciones usadas como vehículo de impregnación de la cepa probiótica $L$. casei en la matriz vegetal de mora de castilla, antes y después del proceso. En ella se observa que el único parámetro que se vió afectado tras el proceso de impregnación fue el $\mathrm{pH}$, el cual presentó una reducción con diferencias estadísticamente significativas $(p<0.05)$, atribuida a la acidez que pudo sumarse al medio tras la inoculación con los microorganismos y a la remoción de ácidos solubles de la fruta, la cual tiene lugar durante la inmersión en la solución y se incrementa con los fenómenos de transferencia de masa ocurridos durante el proceso. Este aspecto no afectará de manera notable la estabilidad de los microorganismos si se considera su tolerancia a la acidez, característica propia de cepas probióticas. En un rango similar de $\mathrm{pH}$, Röble obtuvo resultados semejantes al inocular manzana con 
Lactobacillus rhamnosus sin mayor afectación en los recuentos de UFC/g (Röble et al., 2010); (Ribeiro et al., 2014) describen recuentos mayores en la viabilidad para frutas con valores mayores de $\mathrm{pH}(\mathrm{pH} 4.5-5.2)$ observando un descenso proporcional de la viabilidad con la acidez de la fruta. Estos autores sin embargo justifican la sobrevivencia de los microorganismos a las características físico-químicas de las frutas y a su porosidad principalmente (Ribeiro et al., 2014).

Las viscosidades determinadas para todas las soluciones se hallan en un rango entre $1.2 \mathrm{mPa}$.s y $1.4 \mathrm{mPa}$.s. Estudios de impregnación realizados con soluciones de pectina mostraron que valores por debajo de 10 $\mathrm{mPa}$.s no afectan el proceso de impregnación. Los resultados obtenidos en este estudio están por debajo de este valor, lo cual facilita el flujo de la solución al interior de la fruta una vez restaurada la presión atmosférica tras la IV y minimiza las pérdidas de carga debidas a la fricción de las partículas del fluido entre sí y contra las paredes de los poros (Martínez-Monzó et al., 1998; Betoret et al., 2012).

Los sólidos solubles de las soluciones de impregnación (INU 8.0 \pm 0.5 ; FOS 8.4 \pm 0.5 e INU+ FOS 9.5 \pm 0.67 ), comparadas con las de la mora fresca (MF 8.5 \pm 0.9 ), no presentaron diferencias significativas, lo que asegura un medio isotónico durante los procesos y a su vez da la seguridad de que no existirán mecanismos de transferencia de masa no deseados durante la impregnación.

\section{Determinación de los parámetros de impregnación}

En la tabla 2 se presentan los resultados de los valores promedio y las desviaciones estándar de los parámetros de impregnación obtenidos en el proceso para cada una de las soluciones vehículo, la diferencia de peso de las muestras antes y después del proceso $(\Delta \mathrm{M})$ y la porosidad efectiva $\varepsilon_{\mathrm{e}}$. En la tabla $2, \mathrm{X}_{\mathrm{MF}}=$ Fracción volumétrica de impregnación al final del proceso; $X_{I V}=$ Fracción másica de impregnación al final de la etapa de vacío; $\Delta \mathrm{M}=$ diferencia de masa inicial y final durante el proceso; $\varepsilon_{\mathrm{e}}=$ Porosidad efectiva disponible para el proceso de impregnación

Tabla 2. Parámetros de impregnación teóricos considerados en el sistema

\begin{tabular}{|c|c|c|c|c|c|c|}
\hline \multicolumn{2}{|c|}{ Parámetro } & $\begin{array}{c}\text { Porosidad real } \\
E\left(m^{3} \text { aire } / m^{3}\right. \\
M F)\end{array}$ & $\begin{array}{c}\mathrm{X}_{\mathrm{MF}}{ }^{*} 10^{-2} \\
\left(\mathrm{~m}^{3} \mathrm{sln} / \mathrm{m}^{3}\right. \\
\mathrm{MF})\end{array}$ & $\begin{array}{c}\mathrm{X}_{\mathrm{IV}}^{*} 10^{-3} \\
\text { (Kg prebiótico/Kg } \\
\mathrm{Ml} \text { ) }\end{array}$ & $\Delta \mathrm{M}(\mathrm{g})$ & $\begin{array}{c}\text { Porosidad } \\
\text { aparente } \\
\varepsilon_{\mathrm{e}}\left(\mathrm{m}^{3} \text { aire } / \mathrm{m}^{3} \mathrm{MF}\right)\end{array}$ \\
\hline \multirow{5}{*}{ 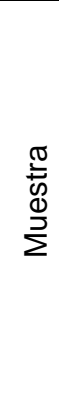 } & Mora fresca & $0.036 \pm 0.01$ & - & - & - & - \\
\hline & Control & - & $2.94 \pm 0.03$ & - & $\begin{array}{c}0.027 \pm \\
0.03\end{array}$ & $0.030 \pm 0.04$ \\
\hline & Inulina & - & $7.69 \pm 0.03$ & $6.658 \pm 0.003$ & $\begin{array}{c}0.068 \pm \\
0.03\end{array}$ & $0.080 \pm 0.04$ \\
\hline & FOS & - & $5.55 \pm 0.02$ & $3.624 \pm 0.004$ & $\begin{array}{c}0.057 \pm \\
0.03\end{array}$ & $0.039 \pm 0.03$ \\
\hline & $\begin{array}{c}\text { Inulina + } \\
\text { FOS }\end{array}$ & - & $7.04 \pm 0.02$ & $5.854 \pm 0.001$ & $\begin{array}{c}0.073 \pm \\
0.01\end{array}$ & $0.074 \pm 0.02$ \\
\hline
\end{tabular}

Los valores obtenidos para la porosidad efectiva $\varepsilon_{e}$ dan una idea del espacio a ser ocupado por la solución vehículo en la matriz estructural. En este caso, los valores de porosidad efectiva y fracción volumétrica están muy cercanos. Con lo cual se podría predecir que la solución vehículo estaría ocupando casi la totalidad del volumen disponible.

En resultados de trabajos realizados con otras frutas se reportaron diferencias entre los dos parámetros mencionados. En estos casos, la porosidad fue mayor que el valor calculado para la fracción volumétrica del líquido de impregnación ( $\mathrm{X}_{\mathrm{MF}}$ ). Estas observaciones fueron justificadas al considerar los fenómenos de deformación de la matriz proteica que ocurren durante el proceso de impregnación, debidos al efecto del diferencial de presiones al interior de los tejidos. De igual manera podrían estar afectando algunas características inherentes a las soluciones de impregnación. En nuestro caso éstas serían descartadas por su carácter isotónico (Fito y Pastor, 1994; Cortés et al., 2007).

Respecto a los valores promedio obtenidos para la fracción volumétrica ocupada por cada uno de los líquidos de impregnación, el mayor valor se obtuvo para inulina, seguida de Inulina + FOS, y por último FOS. Este mismo comportamiento se presentó para la fracción másica de solución, donde se pudo determinar el contenido teórico de prebiótico. Los porcentajes más altos para las muestras impregnadas fueron obtenidos para las soluciones de inulina e inulina+FOS de $0.66 \%$ y $0.58 \%$ respectivamente. Con estos resultados, podemos considerar a las soluciones de inulina, e inulina + FOS como favorables para posteriores investigaciones en alimentos donde la incorporación de fibra prebiótica al interior de una matriz estructural alimenticia sea requerida. 


\section{Caracterización fisicoquímica de las moras después del proceso de impregnación}

En la tabla 3 se presentan los resultados de la caracterización fisicoquímica de las muestras de mora impregnada e impregnada y liofilizada con cada una de las soluciones de impregnación empleadas. Al comparar los resultados de actividad de agua $\mathrm{y}^{\circ}$ Brix de las muestras impregnadas (tabla 3 ) con los obtenidos para la mora fresca $\left(\mathrm{aw}=0.98\right.$; $\left.{ }^{\circ} \mathrm{Brix}=8.5 \pm 0.5\right)$, no se observan diferencias. Esto muestra que el fenómeno de transferencia no se ve afectado por el diferencial de concentraciones tal como se esperaba al usar una solución isotónica en la impregnación. Se considera así, que los cambios en la impregnación solo se relacionan con la diferencia de presiones cuando se trabaja con soluciones isotónicas. El análisis de varianza realizado para las muestras de mora impregnada con las diferentes soluciones, no mostró diferencias estadísticamente significativas ( $p>0.05$ ) por efecto del tratamiento de impregnación en el pH y la actividad del agua. Iguales resultados se obtuvieron para los parámetros evaluados en la mora impregnada y liofilizada. Estos resultados están acordes con el objetivo planteado al usar una solución isotónica de impregnación con la cual se pretende minimizar los fenómenos de transferencia de masa no deseados en el sistema.

Tabla 3. Caracterización físico-química realizada a las muestras de mora impregnada e impregnada liofilizada

\begin{tabular}{|l|l|l|l|l|}
\hline Muestra & Parámetros & Soluciones & \multicolumn{2}{|l|}{} \\
\cline { 3 - 5 } & & Inulina & Inulina+FOS & FOS \\
\hline \multirow{4}{*}{$\begin{array}{l}\text { Mora } \\
\text { impregnada }\end{array}$} & $\mathrm{pH}$ & $3.27 \pm 0.01$ & $3.05 \pm 0.01$ & $3.11 \pm 0.01$ \\
\cline { 2 - 5 } & ${ }^{\circ}$ Brix & $9.0 \pm 0.6$ & $8.7 \pm 0.8$ & $8.5 \pm 0.5$ \\
\cline { 2 - 5 } & $\mathrm{Aw}$ & $0.99 \pm 0.01$ & $0.99 \pm 0.01$ & $0.98 \pm 0.01$ \\
\cline { 2 - 5 } & Humedad & $91.28 \pm 0.02$ & $91.12 \pm 0.05$ & $91.46 \pm 0.02$ \\
\hline \multirow{4}{*}{ Mora liofilizada } & $\mathrm{pH}$ & $4.02 \pm 0.02$ & $4.56 \pm 0.02$ & $4.30 \pm 0.01$ \\
\cline { 2 - 5 } & ${ }^{\circ}$ Brix & $11 \pm 0.5$ & $10 \pm 0.6$ & $10.0 \pm 0.6$ \\
\cline { 2 - 5 } & Aw & $0.33 \pm 0.01$ & $0.34 \pm 0.01$ & $0.39 \pm 0.01$ \\
\cline { 2 - 5 } & Humedad & $3.56 \pm 0.6$ & $4.021 \pm 0.5$ & $3.23 \pm 0.5$ \\
\hline
\end{tabular}

Evaluación de la Viabilidad de Lactobacillus casei en el producto impregnado y liofilizado

La figura 1 muestra los resultados del estudio de viabilidad de $L$. casei en muestras de mora liofilizadas con las diferentes soluciones vehículo y almacenadas a temperatura ambiente $\left(20^{\circ} \mathrm{C}\right)$ durante 40 días. El ANOVA mostró diferencias significativas $(p<0,05)$ en la viabilidad de los microorganismos impregnados en matrices de mora aplicando diferentes soluciones de impregnación con agentes prebióticos. Una prueba de Duncan mostró diferencia entre la muestra control y las muestras impregnadas con las soluciones de agentes prebióticos, después de 40 días de almacenamiento. Ello permitió identificar dos grupos homogéneos claramente diferenciados, uno conformado por las muestras impregnadas con las tres soluciones con prebiótico y otro por la muestra control. La figura 2 muestra los resultados de las medias para cada una de las muestras

El porcentaje de reducción de la viabilidad tras el tratamiento de liofilización fue bajo (alrededor del 32\%) ya en el almacenamiento se observó una paulatina reducción de la viabilidad (UFC/mL) para los microorganismos del grupo control, próxima a una unidad logarítmica cada cinco días de almacenamiento, para una pérdida total de viabilidad de cinco unidades logarítmicas en 40 días de almacenamiento. Resultados reportados por (Ribeiro et al.,2014) tras un proceso de secado a $40^{\circ} \mathrm{C}$ muestran que no se observan recuentos de viables luego de 37 días almacenados a temperatura ambiente, Serna et al., (2015) incorporaron probióticos en cobertura de chocolate para ser utilizada como recubrimiento en barras de cereal, los resultados de viabilidad en esta matriz fueron de $10^{7} \mathrm{UFC} / \mathrm{g}$, al final del proceso de fabricación y consideran que el recuento es bajo debido a que los microorganismos aún deben sobrevivir al proceso de almacenamiento y a los jugos gástricos e intestinales del consumidor. En nuestro caso, el porcentaje de reducción de viabilidad durante el tratamiento de secado y posterior almacenamiento fue bajo, probablemente debido al efecto termoprotector del agente prebiótico. Capela et al., (2006) reportan que durante el procesamiento y almacenamiento de alimentos probióticos, variables como el contenido de oxígeno, altas temperaturas, $\mathrm{pH}$, alta actividad de agua y elevada concentración de solutos, pueden afectar la viabilidad de los microorganismos probióticos; y sugieren que el uso de agentes prebióticos favorece la viabilidad de los microorganismos probióticos en diferentes ambientes y actúan como agentes termoprotectores durante la liofilización, destacando entre ellos la inulina. 


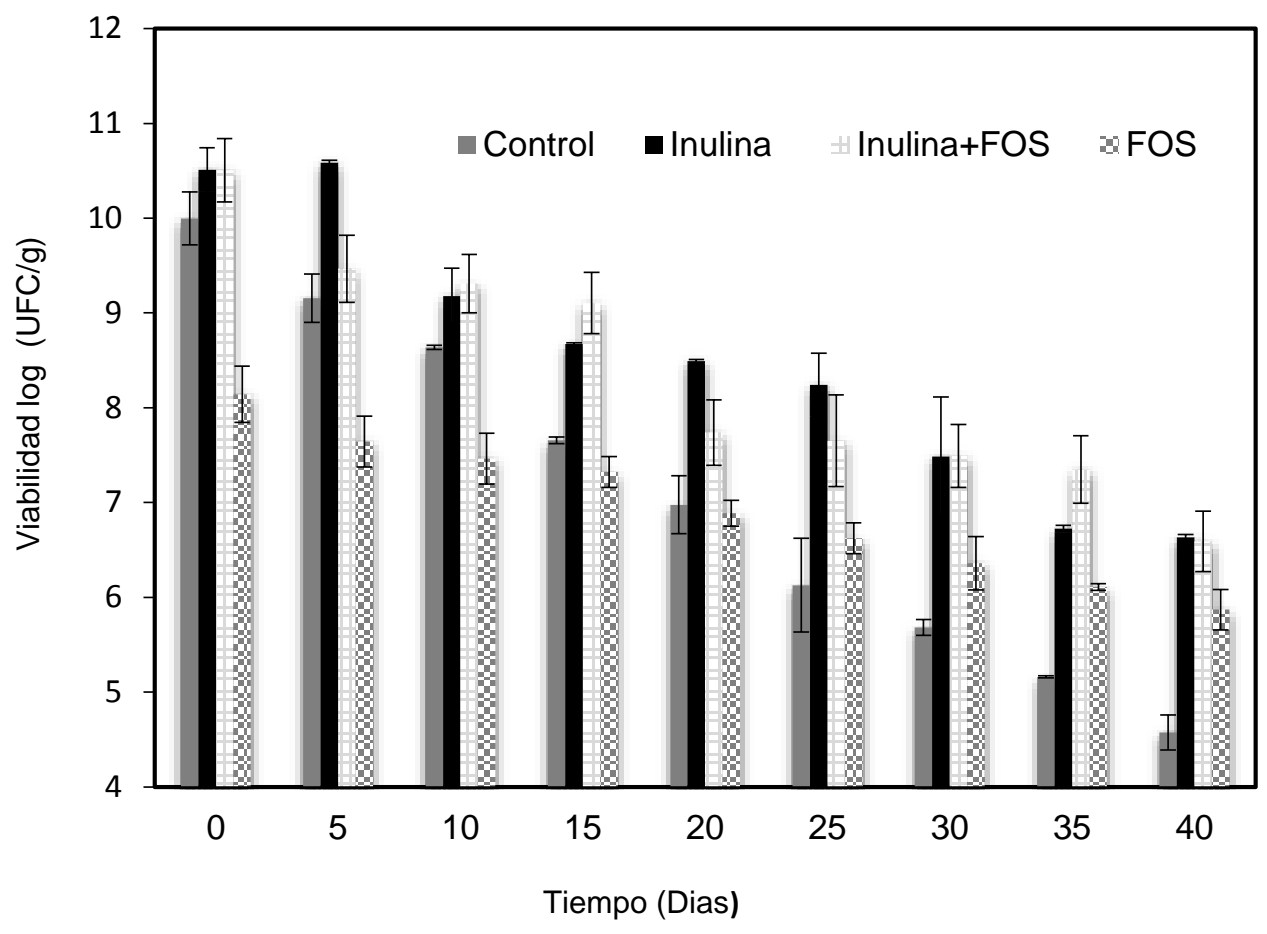

Fig. 1. Viabilidad del microorganismo durante el almacenamiento

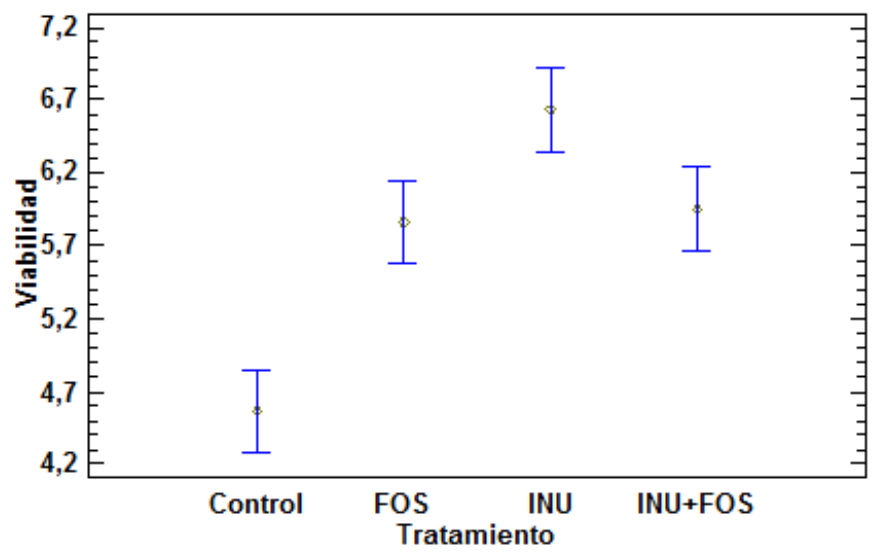

Fig. 2. Gráfico de medias de viabilidad para L. casei después de 40 días de almacenamiento

Para el caso de las muestras impregnadas con solución prebiótica, esta reducción fue solo de aproximadamente tres unidades logarítmicas durante el periodo evaluado. Este resultado permite predecir que en todos los casos, partiendo de una viabilidad superior a $10^{9} \mathrm{UFC} / \mathrm{gr}$ en la muestra, luego de 40 días de almacenamiento a temperatura de $20 \pm 2{ }^{\circ} \mathrm{C}$, el alimento mantendrá sus características probióticas y por tanto su potencial simbiótico. Estos resultados corroboran los datos reportados por algunos autores con respecto al efecto favorable de la presencia de agentes prebióticos sobre la viabilidad de microorganismos probióticos (Gibson et al., 2004; Capela et al., 2006). Adicionalmente, tal como reportan Holzapfel et al. (2002), este alimento, con la presencia y actividad sinérgica de prebióticos y probióticos en su estructura, favorecería la promoción de la salud tras su consumo regular.

\section{Análisis microscópico}

Se realizó una valoración de la presencia de la cepa inoculada al interior de la matriz porosa de láminas de mora de castilla impregnadas y liofilizadas, mediante la aplicación de la técnica de microscopía electrónica de barrido. Las figuras $3 a$ y $3 \mathrm{~b}$ presentan las imágenes de un segmento de la estructura porosa de la mora y la presencia de aglomerados de BAL probióticas en su forma típica ovalada de bordes redondeados. Otros autores han obtenido resultados similares con la aplicación de la técnica de impregnación a vacío de 
microorganismos en la matriz porosa de manzana aplicando zumos de fruta inoculada como vehículo de incorporación (Puente et al., 2009, Betoret et al., 2003 ).
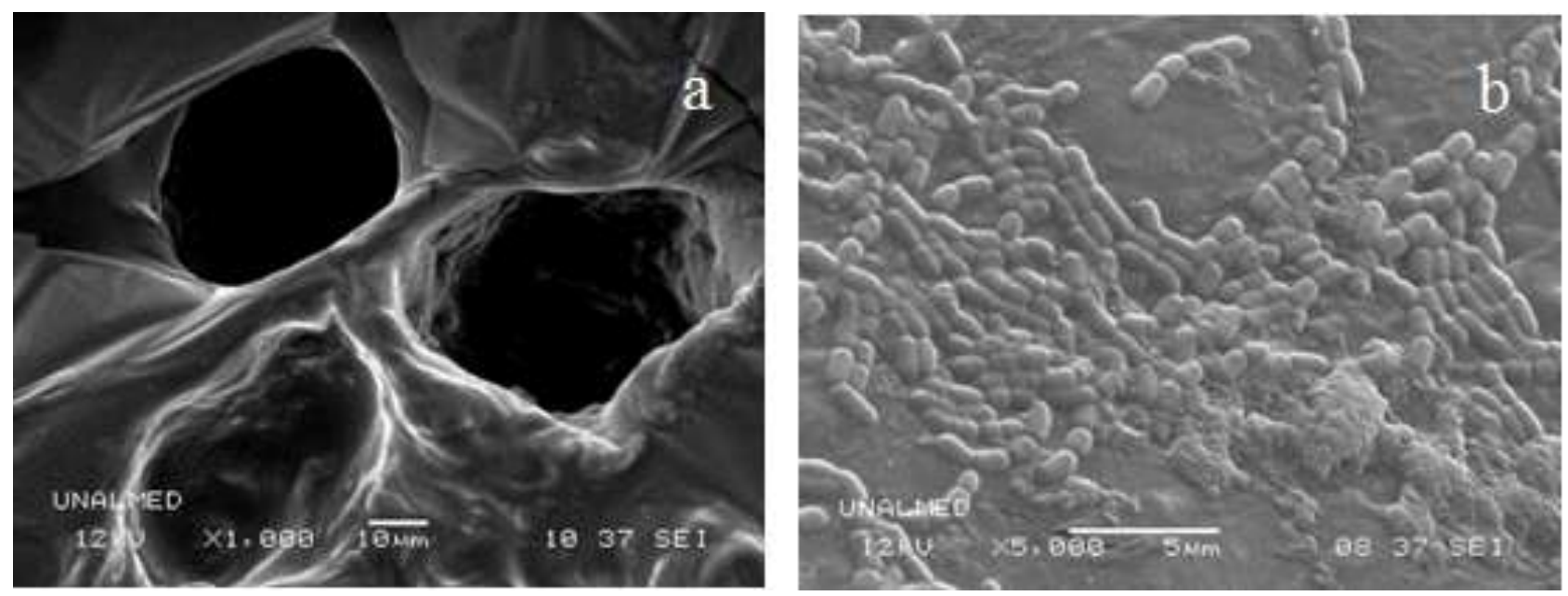

Fig. 3. Micrografías SEM a) Matriz estructural de mora de castilla. b) L. casei impregnados en la superficie del tejido de mora.

\section{Análisis sensorial}

Las figuras $4 \mathrm{a}$ y $4 \mathrm{~b}$ muestran los resultados de la prueba de aceptabilidad realizada con un panel de 33 jueces no entrenados consumidores habituales de yogurt y cereales en el desayuno.
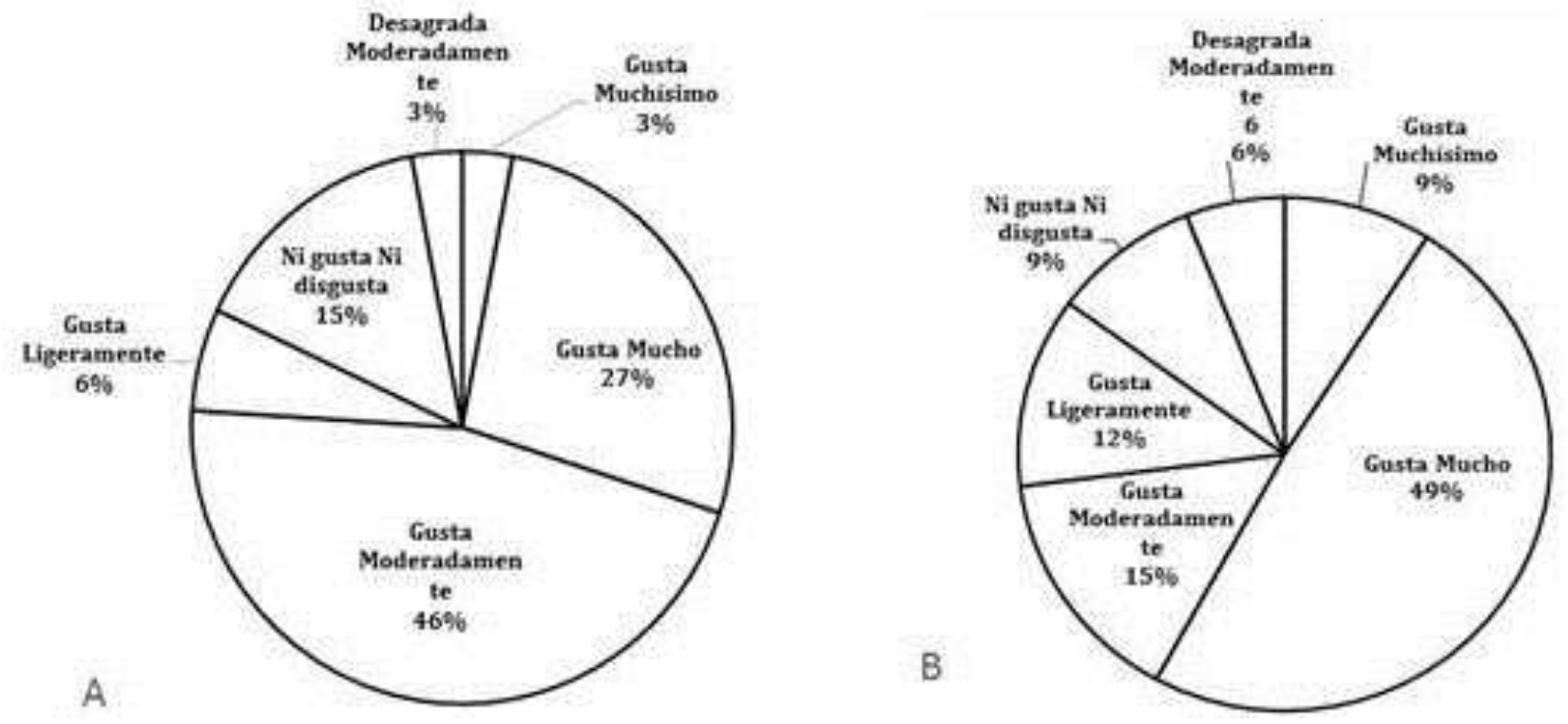

Fig. 3. Resultados de las pruebas de aceptabilidad, a) yogurt, b) cereal

En las muestras de mora impregnada y liofilizada, incorporados en yogurt y en cereal con frutos secos comerciales; se observa que para la muestra con yogurt los porcentajes más altos de aceptación fueron "gusta moderadamente" y "gusta mucho" con un $46 \%$ y $27 \%$ en las respuestas respectivamente, seguida de un $15 \%$ para la respuesta "Ni me gusta ni disgusta". En el caso de las muestras incorporadas a una mezcla de cereal, un $9 \%$ de las respuestas reporto un "me gusta muchísimo" mientras que el $49 \%$ de las respuestas se reportó en el rango de la escala "Me gusta mucho". Los resultados permiten afirmar que el producto -láminas de mora bio-impregnadas con L. casei y agentes prebióticos- fue aceptado y bien valorado por los consumidores potenciales al ser incorporado como ingrediente de alimentos de consumo habitual. 


\section{CONCLUSIONES}

En el presente trabajo se obtuvo un alimento funcional probiótico potencialmente simbiótico no lácteo, mediante la incorporación de soluciones de impregnación inoculadas con la bacteria $L$. casei en la matriz de mora de castilla con una significativa aceptación por el consumidor tras su incorporación como ingrediente alimenticio. Se observó una mejora significativa en la viabilidad del microorganismo probiótico incorporado bajo el efecto de la inulina en la mezcla de impregnación dejando en evidencia su efecto termoprotector e inductor en la viabilidad de L. casei. Este alimento, con cualidades simbióticas, tras el efecto sinérgico del prebiótico y el probiótico incorporado, aportará, bajo un consumo frecuente, beneficios en la salud integral del consumidor. Con este trabajo se hace un aporte de valor a la cadena agroindustrial de la mora de castilla aplicable a otros productos con características estructurales similares.

\section{REFERENCIAS}

Aljewicz, M., Cichosz, G., The effect of probiotic Lactobacillus rhamnosus HN001 on the in vitro availability of minerals from cheeses and cheese-like products, LWT - Food Science and Technology: 60, 841-847 (2015)

A.O.A, C. Method 934.06. Moisture in dried fruits. In: Official method of Analysis of AOAC International (1999)

Andrés, Bello., A.C. De Jesús., P. García-Segovia., J. Martínez-Monzó J y Pagán-Moreno M. J, Vacuum impregnation as a tool to introduce biopreservatives in gilthead sea bream fillets (Sparus aurata), LWT - Food Science and Technology: 60(2), 758-765 (2015)

Anzaldua, A, La evaluación sensorial de los alimentos en la teoria y la práctica, Editorial Acribia, Zaragoza España (1994)

Betoret, E., N. Betoret., A. Arilla., M. Bennàr., P. Codoñer y P. Fito, No invasive methodology to produce a probiotic low humid apple snack with potential effect against Helicobacter pylori, Journal of Food Engineering :110, 289-293 (2012)

Betoret, N. y otros siete autores, Development of probiotic-enriched dried fruits by vacuum impregnation, Journal of food Engineering: 56(2), 273-277 (2003)

Capela, P., T. K. Hay y N. P. Shah, Effect of cryoprotectants, prebiotics and microencapsulation on survival of probiotic organisms in yoghurt and freeza-dried yoghurt, Food Research Intenational: 39, $203-211$ (2006)

Cortès, R., M., L. F. Guardiola y R. Pacheco, Aplicación de la ingeniería ee matrices en la fortificación de mango (Var. Tommy Atkins) con calcio, DYNA :74, 19-26 (2007)

Champagne, C. P., R. P. Ross, M. Saarela, K. F. Hanen y D. Charalampopoulos, Recomendations for the viability asessment of probiotics as concentrated cultures in a food matrices, Internationa Journal of Food Microbiology: 149, 185-193 (2011)

De Vrese, M. y J. Schrezenmeir, Probiotics, prebiotics, and synbiotics, Advances in Biochemical Engineering Biotechnology : 111, 1-66 (2008)

Fito, P.y otros siete autores, Vacuum impregnation and osmotic dehydration in matrix engineering: Application in functional fresh food development, Journal of Food Engineering: 49(2-3), 175-183 (2001)

Fito, P. y R. Pastor, n some diffusional mechanism occurring Vacuum Osmotic Dehydration (VOD), Journal of Food Engineering: 51(21), 513-519 (1994)

Gibson, G. R., H. M. Probert, J. V. Loo, R. A. Rastall y M. B. Roberfroid, Dietary modulation of the human colonic microbiota: updating the concept of prebiotics, Nutrition Research Reviews: 17(2), 259-275 (2004)

Gras, M. L., D. Vidal, N. Betoret, A. Chiralt y P. Fito, Calcium fortification of vegetables by vacuum impregnation, interactions with cellukar matrix, Journal of Food Engineering: 56, 279-284 (2003)

Holzapfel, W. H. y U. Schillinger , Introduction to pre- and probiotics, Food Research International: 35, 109116 (2002)

Krasaekoopt, W., Watcharapoka, S., Effect of addition of inulin and galactooligosaccharide on the survival of microencapsulated probiotics in alginate beads coated with chitosan in simulated digestive system, yogurt and fruit juice, LWT - Food Science and Technology: 57, 761-766 (2014)

Martínez-Monzó, J., N. Martínez-Navarrete, A. Chiralt y P. Fito , Mechanical and structural changes in apple (var. Granny Smith) due to vacuum impregnation with cryoprotectants, Journal of Food Science: 63(3), 499503 (1998) 
Mertz, C., Anne-Laure G., Gunata, Z., Alter, P., Dhuique-Mayer,C., Vaillant, F., Pérez A.M., Perez Ruales, Y., and Brat, P., Phenolic compounds, carotenoids and antioxidant activity of three tropical fruits, Journal of Food Composition and Analysis: 22, 381-387 (2009)

Puente, L., N. Betoret y M. Cortes, Evolution of probiotic content and color of apples impregnated with lactic acid bacteria, Vitae : 16, 297-303 (2009)

Rajam, R., Anandharamakrishnan, C., Spray freeze drying method for microencapsulation of Lactobacillus plantarum, Journal of Food Engineering: 166, 95-103 (2015)

Rêgo, A., R. Freixo, J. Silva, Gibbs, P., A. M. M. B. Morais y P. Teixeira, A functional dried fruit matrix incorporated with probiotic strains: lactobacillus plantarum and lactobacillus kefir, Focusing on Modern Food Industry: 2(3), 138-143 (2013)

Ribeiro, C., R. Freixo, J. Silva, P. Gibbs, B. Moraisa y P. Teixeira, Dried Fruit Matrices Incorporated with a Probiotic Strain of Lactobacillus plantarum, International Journal of Food Studies :3, 69-73 (2014)

Roberfroid, M. B, Prebiotics and probiotics: Are they functionals foods? , American Journal Clinical Nutirition :71(6), 1682-1687 (2000)

Röble, C., M. A. E. Auty, N. Brunton, R. T. Gormley y F. Butler, Evaluation of fresh-cut apple slices with probiotic bacteria, Innovative Food Science and Emerging Technologies : 11, 203-209 (2010)

Sarmiento, L. A, Alimentos funcionales, una nueva alternativa de alimentación, Revista Orinoquia, Universidad de los Llanos. Villavicencia, Meta Colombia : 10, 16-23 (2006)

Serna-Cock, L., Angulo-López, J. E, A. Ayala-Aponte, A., Barras de Cereal como Matriz Sólida para la Incorporación de Microorganismos Probióticos, doi: 10.4067/S0718-07642015000200005, Información Tecnológica: 26(2), 29-40 (2015)

Tripathi, M. K. y S. K. Giri, Probiotic functional foods: Survival of probiotics during processing and storage , Journal of Functional Foods : 9 , 225-241 (2014)

Ursachia, C., R. Segalb y C. Muresana, Vacuum Impregnation Viability of Some Fruits, Journal of Agroalimentary Processes and Technologies : 15(2), 316-319 (2009)

Vasiljevic, T. y N. P. Shah, Probiotics_From Metchnikoff to bioactives, International Dairy Journal : 18(7), 714-728 (2008)

Zapata, I.C., Sepúlveda-Valencia, U., Rojano, B., Efecto del Tiempo de Almacenamiento sobre las Propiedades Fisicoquímicas, Probióticas y Antioxidantes de Yogurt, doi: 10.4067/S071807642015000200004, Información Tecnológica: 26(2), 17-28 (2015) 